\title{
Full-field interferometric confocal microscopy using a VCSEL array
}

\author{
Brandon Redding, ${ }^{1, *}$ Yaron Bromberg, ${ }^{1}$ Michael A. Choma, ${ }^{2,3}$ and Hui Cao ${ }^{1,4}$ \\ ${ }^{1}$ Department of Applied Physics, Yale University, New Haven, Connecticut 06520, USA \\ ${ }^{2}$ Department of Diagnostic Radiology, Yale School of Medicine, New Haven, Connecticut 06520, USA \\ ${ }^{3}$ Department of Biomedical Engineering, Yale University, New Haven, Connecticut 06520, USA \\ ${ }^{4}$ e-mail: hui.cao@yale.edu \\ *Corresponding author: brandon.redding@yale.edu
}

Received May 1, 2014; accepted June 6, 2014;

posted June 24, 2014 (Doc. ID 211257); published July 24, 2014

\begin{abstract}
We present an interferometric confocal microscope using an array of 1200 vertical cavity surface emitting lasers (VCSELs) coupled to a multimode fiber. Spatial coherence gating provides $\sim 18,000$ continuous virtual pinholes, allowing an entire en face plane to be imaged in a snapshot. This approach maintains the same optical sectioning as a scanning confocal microscope without moving parts, while the high power of the VCSEL array $(\sim 5 \mathrm{~mW}$ per laser) enables high-speed image acquisition with integration times as short as $100 \mu$ s. Interferometric detection also recovers the phase of the image, enabling quantitative phase measurements and improving the contrast when imaging phase objects. (C) 2014 Optical Society of America

OCIS codes: (180.1790) Confocal microscopy; (110.4980) Partial coherence in imaging; (180.3170) Interference microscopy.

http://dx.doi.org/10.1364/OL.39.004446
\end{abstract}

Confocal microscopy combines high resolution with improved contrast and optical sectioning, making it an invaluable tool in developmental biology, clinical medicine, and optical metrology [1,2]. However, traditional confocal microscopes rely on raster scanning, which limits image acquisition speed and increases system complexity. With typical frame rates of a few hertz for $1000 \times 1000$ pixel frames, scanning confocal systems are susceptible to motion artifacts and are poorly suited for the study of dynamic samples or for use in vivo. While video-rate confocal microscopes have been demonstrated using very high-speed scanning $[3,4]$, the complexity required to achieve such high scan rates has limited their adoption. Given a fixed lateral scan rate, the image acquisition speed can also be improved through parallelization. The most common approach to parallelization is through the use of an array of spatially separated pinholes (i.e., a Nipkow disk) [5]; however, this approach has obvious limitations, since the pinholes must be sufficiently separated to prevent cross talk [ㅁ,7]. Researchers have also proposed sacrificing confocality in one dimension (i.e., line-scan confocal microscopy [8-10]) to improve imaging speed, but cross talk limits this approach to weakly scattering samples [11]. Spectral encoding can provide parallelization in one dimension without cross talk by using a grating to map different wavelengths to a line on the sample; however, scanning in the second dimension is still required to form an image [12].

An alternative approach to completely parallelize confocal image acquisition is to combine interferometric detection with spatial coherence gating [13-17]. In this approach, each spatial mode (defined by the spatial coherence area) acts as a virtual pinhole, since interference only occurs for light from a single spatial mode. Unlike physical pinholes, these virtual pinholes do not require physical separation to avoid cross talk, enabling parallel acquisition of an entire en face plane in a single snapshot without scanning. Although this type of microscope cannot be used for fluorescence imaging, it has the potential for high-speed, large-area reflectance imaging with confocal resolution and sectioning [14]. However, the main advantage of parallelization-faster image acquisition-has thus far been mitigated by the lack of an appropriate light source. Traditional low-spatialcoherence sources (e.g., thermal sources or lightemitting diodes) lack sufficient power per mode for high-speed imaging, and methods to reduce the spatial coherence of lasers (e.g., rotating diffusers) require relatively long integration times to achieve sufficiently low spatial coherence.

In this work, we use a recently developed vertical cavity surface emitting laser (VCSEL) array [18], which combines high power per mode with low spatial coherence, to demonstrate full-field confocal image acquisition with integration times as short as $100 \mu \mathrm{s}$. The VCSEL array consists of $\sim 1200$ mutually incoherent lasers providing $\sim 6 \mathrm{~W}$ of total power $(\sim 5 \mathrm{~mW}$ per laser $)$ at $\lambda=808 \mathrm{~nm}$. Interferometric detection is achieved using an off-axis holography technique, enabling parallel acquisition of image information from 18,000 continuous virtual pinholes. The microscope then provides en face images with a $210 \mu \mathrm{m} \times 280 \mu \mathrm{m}$ field of view, $\sim 2 \mu \mathrm{m}$ lateral resolution, and $\sim 8 \mu \mathrm{m}$ axial resolution in a single shot.

A schematic of the full-field confocal microscope is shown in Fig. 1. A microscope image of the VCSEL array (Princeton Optronics PCW-CE-4-W0808) is shown in Fig. 1(c) and an image of the array lasing is shown in Fig. 1(b). Each laser had a bandwidth of $\sim 0.1 \mathrm{~nm}$ and the combined bandwidth of all 1200 lasers was $\sim 1 \mathrm{~nm}$. The lasers are each $\sim 15 \mu \mathrm{m}$ in diameter and arranged periodically in a $2 \mathrm{D}$ triangular array with a center-tocenter spacing of $\sim 44 \mu \mathrm{m}$. A step index multimode fiber with NA $=0.48$ and a core diameter of $600 \mu \mathrm{m}$ was used to collect the VCSEL array emission through a fiber collimator and deliver it to the microscope. Due to the relatively small divergence angle of the VCSEL array $\left(\sim 10^{\circ}\right)$, 
(a) Full-Field Interferometric Confocal Microscope

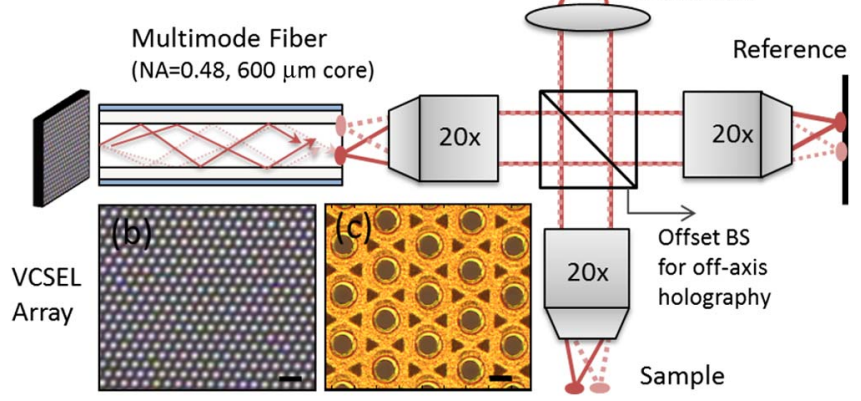

Fig. 1. (a) Schematic of the full-field confocal microscope. (b) Top view of the VCSEL array while lasing-each dot is a separate laser. The scale bar is $100 \mu \mathrm{m}$. (c) Magnified view of the VCSEL array-each circle is an independent laser. The scale bar is $20 \mu \mathrm{m}$. The VCSEL array was coupled to a $1 \mathrm{~m}$ long multimode fiber, which scrambled the modes, providing a uniform illumination source. The emission leaving the multimode fiber was imaged using $20 \times(\mathrm{NA}=0.4)$ objectives onto the sample and reference arms through a beam splitter. The beam splitter was offset from the optical axis of the sample arm in order to introduce interference fringes in the detected signal. A charge-coupled device (CCD) camera was then used to record the interference pattern.

the emission was easily collected with the multimode fiber. Each laser in the VCSEL array excited a different distribution of spatial modes in the fiber, resulting in distinct speckle patterns at the end of the multimode fiber. Since each of the $\sim 1200$ lasers is mutually incoherent, their speckle patterns are uncorrelated and add in intensity, providing a high-power light source with low spatial coherence. Although multimode fibers can also reduce the spatial coherence of an individual laser if the temporal coherence is low [19], this effect is expected to be weak in our case due to the relatively narrow linewidth $(\sim 0.1 \mathrm{~nm})$ of the individual lasers in the VCSEL array and the short length of the multimode fiber $(1 \mathrm{~m})$. Nonetheless, the speckle contrast at the end of the fiber was reduced to $N^{-1 / 2}$, where $N$ is the number of uncorrelated speckle patterns. In this case, $N \sim 2 \times 1200$, where the factor of 2 accounts for each laser producing speckle patterns at the end of the fiber with orthogonal polarizations, which do not interfere and add in intensity. The speckle contrast was, therefore, reduced to $\sim 0.02$, below the level of intensity variations humans can perceive [20].

The effective low spatial coherence of the VCSEL array can also be understood in comparison with the sources used, for example, in Refs. [14-16], which consisted of a conventional spatially coherent laser passed through a rotating diffuser. The rotating diffuser caused the speckle patterns illuminating the sample and reference to change over time. In those implementations, a relatively long integration time was then required to average over many diffuser positions, thereby reducing the speckle contrast and eliminating cross talk in the detected interferometric signal. In our case, the 1200 lasers each produced a distinct speckle pattern, analogous to the speckle patterns produced by different diffuser positions. However, by using the VCSEL array, averaging over many speckle patterns was performed in parallel. We need only integrate longer than the temporal coherence of the modes ( $\sim 10 \mathrm{ps}$ based on the $\sim 0.1 \mathrm{~nm}$ laser linewidth) to average out speckle. Thus, this approach has the potential for much faster imaging, and could also be used in applications that rely on very short integration times such as stroboscopic imaging [21] for which the rotating diffuser approach is particularly unsuited.

Although the VCSEL array could have been coupled directly into the microscope, the multimode fiber simplified the alignment and ensured spatially uniform illumination of the sample and reference arms. As shown in Fig. 1, the core of the multimode fiber was imaged through $20 \times$ objectives $(\mathrm{NA}=0.4)$ onto the reference and sample arms. By placing a mirror on the reference arm, an image of the multimode fiber facet was produced on the CCD camera (Allied Vision Manta G-125) through a tube lens $(f=15 \mathrm{~cm})$. Light scattered by the sample was also imaged onto the CCD camera and the confocal signal was then encoded in the interference between the sample and reference arms. To enable single-shot detection without phase-stepping, we used an off-axis holography detection scheme. To do this, we offset the beam splitter, which introduced a phase tilt between the reference and sample arm images, resulting in interference fringes.

A typical unprocessed image recorded by placing a U.S. Air Force resolution target at the focal plane of the sample arm is shown in Fig. 2(a). A magnified view of the high-contrast interference fringes is shown in Fig. 2(e). The confocal image was then extracted by applying a Hilbert filter in phase space. The spatial Fourier transform of the unprocessed image is shown in Fig. 2(b). We applied a Hann window to select the region of frequency space indicated by the black circle and moved this region to the center before applying an inverse Fourier transform. The amplitude and phase extracted from the inverse Fourier transform are shown in Figs. 2(c) and 2 (d), respectively. As shown in Fig. 2 (f), the smallest features of the Air Force chart $(2.19 \mu \mathrm{m}$ lines) were clearly resolved.

The high power per laser of the VCSEL array, combined with the low spatial coherence, which precluded the need for averaging over speckle patterns in time, enabled integration times as short as $100 \mu \mathrm{s}$, the minimum integration time on the camera. Even at such short integration times, the VCSEL array was attenuated to avoid saturating the camera. Thus, we expect that this approach could enable high-speed imaging of dynamic behavior with confocal resolution and sectioning. Moreover, the single-shot image acquisition avoids the effect of motion artifacts and may be used for stroboscopic imaging by running the VCSEL array in pulsed mode.

In contrast to a standard scanning confocal microscope, which records only the intensity, the interferometric confocal system presented here also records the phase of the field scattered by the sample. In the case of an Air Force chart, which consists of Chrome on glass, this phase information allows us to extract the step height, $h$, of the Air Force chart features as $h=\varphi[\lambda /(4 \pi)]$, where $\lambda$ is the illumination wavelength $(808 \mathrm{~nm})$ and $\varphi$ is the relative phase difference between the Chrome and the glass features of the phase image shown in Fig. 2(d). Note that what we measure is the phase of the field returning from the 
(a) Unprocessed Image

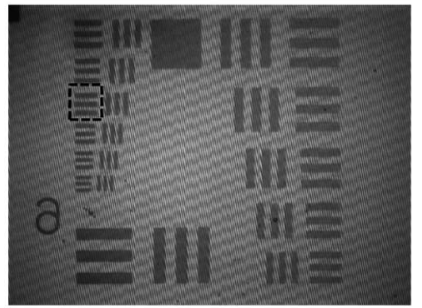

(c) Amplitude Image
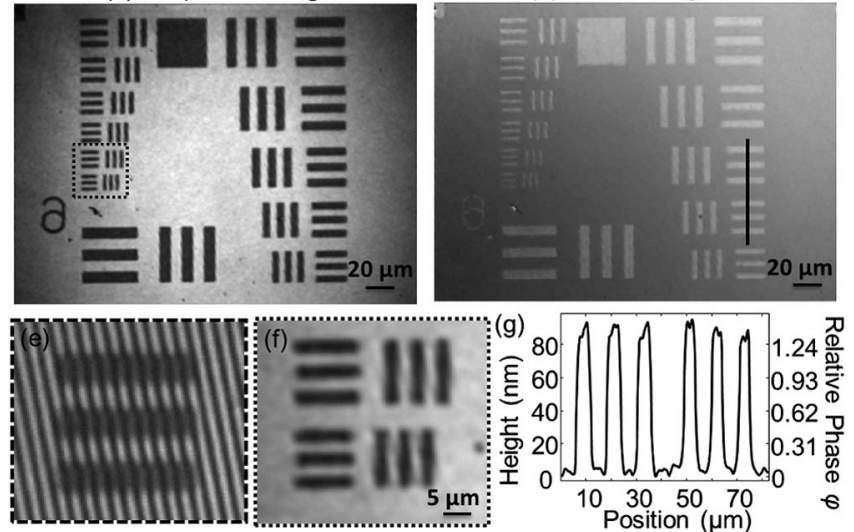

Fig. 2. (a) Unprocessed interference pattern measured on the CCD camera shown in Fig. 1. High-contrast fringes are visible across the image. (b) Spatial Fourier transform of the image in (a). A Hann window, indicated by the black circle, was used to select the off-axis component. This component was then shifted back to the center of Fourier space. The (c) amplitude and (d) phase of the field from the sample are recovered after inverse Fourier transform of the filtered image in (b). (e) Magnified view of the region indicated in (a) showing high-contrast interference fringes. (f) Magnified view of the region indicated in (c); features separated by $2.19 \mu \mathrm{m}$ are clearly distinguishable. (g) Step height of the Air Force chart features extracted from the phase image along the cross-section line shown in (d).

sample, and thus, to accurately extract the step height, we need to account for the phase delay introduced by reflections from different surfaces. In particular, the reflection from the air-Chrome interface introduces an additional phase delay of 0.32 radians compared with the reflection from the air-glass interface, due to the complex index of refraction of Chrome $(n \sim 3.18+3 i$ at $\lambda=808 \mathrm{~nm}$ ). After accounting for this factor, the step height of the Air Force chart can be measured accurately. In Fig. 2(g), we show the extracted step height measured across the group 6 features of the Air Force chart, indicating an $80 \mathrm{~nm}$ step. This step height was confirmed using a mechanical profilometer (Alpha-Step IQ Surface Profiler).

As in confocal systems using a physical pinhole, the interferometric confocal system shown here provides optical sectioning. To measure the axial point spread function (PSF), we placed a mirror in the sample arm and scanned its position through the focal plane of the objective and recorded the intensity of the confocal image. As shown in Fig. 3, the interferometric confocal system shows a sharp axial response with a full-width at halfmaximum (FWHM) of $\sim 8 \mu \mathrm{m}$. Based on the $0.4 \mathrm{NA}$ of the microscope objectives, we would expect the FWHM axial PSF to be $\sim 1.26 \lambda / \mathrm{NA}^{2}=6.3 \mu \mathrm{m}[1]$. The slight degradation in the observed axial resolution is a result of
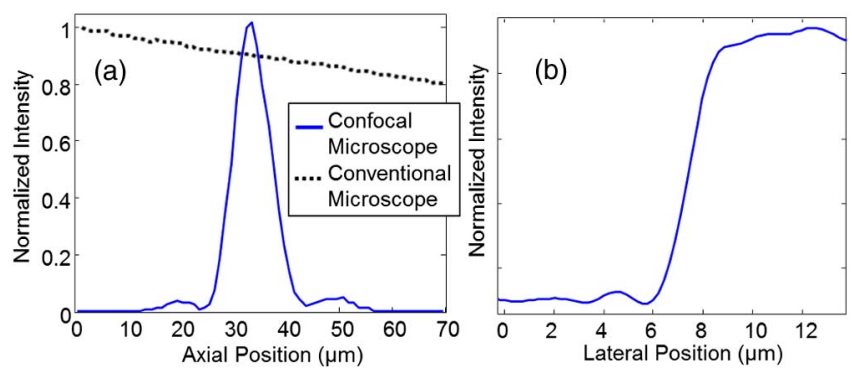

Fig. 3. (a) Axial PSF was recorded by scanning a mirror through the focal plane and recording the intensity of the confocal image as a function of the mirror position. The confocal microscope shows a sharp axial response with FWHM $\sim 8 \mu \mathrm{m}$, compared with the conventional microscope in which the recorded intensity decays slowly with defocus. (b) The lateral PSF was estimated by examining the sharpness of the edge in the image of the resolution chart shown in Fig. 2(c). The half-width at half-maximum is $\sim 1.2 \mu \mathrm{m}$.

imposing the off-axis interference fringes by offsetting the beam splitter. This had the secondary effect of underfilling the back aperture of the objective, thereby reducing the effective NA. Based on the axial PSF observed, the effective NA is $\sim 0.36$. The full resolution of the objectives could be achieved by phase-stepping on the reference arm, rather than using an off-axis holography approach to recover the sample field. Alternately, adding a diffraction grating to one arm could be used to introduce high-spatial-frequency fringes without sacrificing resolution while maintaining single-shot image acquisition $[16,22]$. We also repeated this experiment while blocking the sample arm to provide a comparison with a conventional, noninterferometric wide-field microscope. Although the conventional microscope image blurs with defocus, the total intensity recorded as the mirror is scanned through the focal plane remains relatively constant.

We also estimated the lateral resolution by examining the image intensity across the edge of the features in the Air Force chart shown in Fig. 2(c). As shown in Fig. 3(b), intensity showed a sharp increase across the feature edge, with a half-width at half-maximum of $\sim 1.2 \mu \mathrm{m}$. In the current implementation, the lateral resolution was limited by the off-axis holography approach and could be improved to reach the diffraction limit of the microscope objectives $(0.88 \lambda /(2 \mathrm{NA}) \sim 0.9 \mu \mathrm{m}$ [1] $)$ by phasestepping on the reference arm. The lateral resolution of the microscope also dictates the effective speckle size on the sample and hence, the virtual pinhole size. The total number of virtual pinholes is then given by the field of view divided by the effective speckle size (i.e., a circle with radius $\sim 1 \mu \mathrm{m}$ ). Since the field of view of the image was $210 \mu \mathrm{m} \times 280 \mu \mathrm{m}$, we were able to acquire en face confocal images with the equivalent of $\sim 18,000$ virtual pinholes in parallel. Note that the number of virtual pinholes is not limited to the 1200 lasers in the VCSEL array, since these lasers are used to eliminate cross talk through averaging, rather than as independent imaging channels. This dramatically reduces the required source complexity (i.e., the number of lasers in the array) while allowing massively parallel full-field imaging. In general, we expect the number of virtual pinholes to be limited 

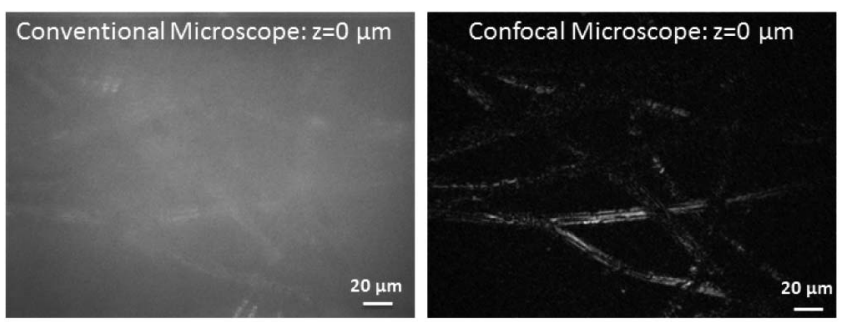

Conventional Microscope: $\mathrm{z}=28 \mathrm{~mm}$
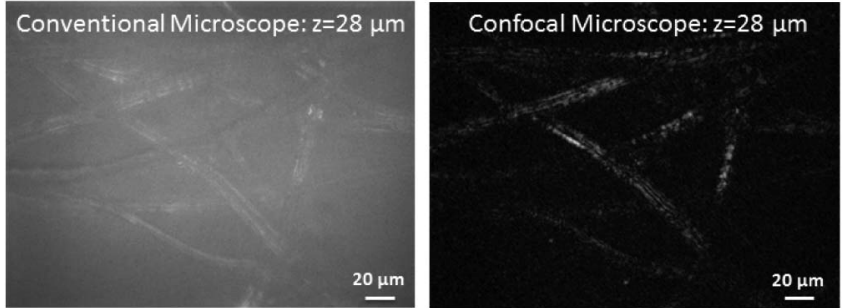

Fig. 4. Images of lens paper at two focal planes separated by $28 \mu \mathrm{m}$. The conventional microscope images were recorded by blocking the reference arm. Unlike the conventional microscope, the optical sectioning ability of the confocal microscope enables imaging of different planes while rejecting out-of-plane light. (Media 1) The supplementary video shows the conventional microscope and confocal images as the lens paper is scanned through the focal plane.

by the number of resolvable elements provided by the microscope objective, whereas the number of lasers required to eliminate cross talk will depend on the degree of scattering in the sample, the required speckle suppression, as well as aberrations in the imaging system. Moreover, the parallel crosstalk suppression provided by the VCSEL array could be combined with temporal averaging over different speckle patterns (introduced by a rotating diffuser or simply by shaking the multimode fiber to change the mode coupling conditions). This could enable averaging over many more speckle patterns to further suppress cross talk at the cost of increased integration time.

To demonstrate the optical sectioning capabilities of the confocal microscope, we recorded a series of images at different planes by scanning a piece of lens paper across the focal plane. The lens paper consists of a threedimensional structure of threads. In Fig. 4, we have shown images recorded at two different planes with the confocal microscope along with images taken with a conventional wide-field microscope (i.e., with the reference arm blocked). In the confocal images, the threads of the lens paper in the focal plane are clearly visible while scattered light from out-of-plane threads is rejected. However, in the conventional microscope image, the contribution from out-of-plane threads introduces a strong blurry background, which reduces the image contrast. A movie showing 40 frames collected as the lens paper was scanned across the focal plane is provided in Media 1.

In summary, we have demonstrated a full-field interferometric confocal microscope using spatial coherence gating to acquire single-shot en face images. Illumination was provided by a VCSEL array, which combined high power per mode with low spatial coherence, enabling high-speed parallel image acquisition. The confocal system collected $210 \mu \mathrm{m} \times 280 \mu \mathrm{m}$ field of view images with $\sim 2 \mu \mathrm{m}$ lateral and $\sim 8 \mu \mathrm{m}$ axial resolution at integration times as short as $100 \mu \mathrm{s}$. The same system could be adapted to provide higher resolution imaging using higher NA microscope objectives. In addition, interferometric detection provides access to phase information, enabling quantitative phase measurements, enhancedcontrast imaging of phase objects, or Doppler flow measurements.

This work was supported by the National Science Foundation under Grant No. ECCS-1128542 and the National Institutes of Health under Grant No. 1R21EB016163-01A1.

\section{References}

1. R. H. Webb, Rep. Prog. Phys. 59, 427 (1996).

2. G. S. Kino and T. R. Corle, Confocal Scanning Optical Microscopy and Related Imaging Systems (Academic, 1996).

3. M. Rajadhyaksha, R. R. Anderson, and R. H. Webb, Appl. Opt. 38, 2105 (1999).

4. J. T. C. Liu, M. J. Mandella, H. Ra, L. K. Wong, O. Solgaard, G. S. Kino, W. Piyawattanametha, C. H. Contag, and T. D. Wang, Opt. Lett. 32, 256 (2007).

5. M. Petráň, M. Hadravský, M. D. Egger, and R. Galambos, J. Opt. Soc. Am. 58, 661 (1968).

6. A. Egner, V. Andresen, and S. W. Hell, J. Microsc. 206, 24 (2002)

7. T. Shimozawa, K. Yamagata, T. Kondo, S. Hayashi, A. Shitamukai, D. Konno, F. Matsuzaki, J. Takayama, S. Onami, H. Nakayama, Y. Kosugi, T. M. Watanabe, K. Fujita, and Y. Mimori-Kiyosue, Proc. Natl. Acad. Sci. USA 110, 3399 (2013).

8. P. J. Dwyer, C. A. DiMarzio, and M. Rajadhyaksha, Appl. Opt. 46, 1843 (2007).

9. B. Larson, S. Abeytunge, and M. Rajadhyaksha, Biomed. Opt. Express 2, 2055 (2011).

10. K.-B. Im, S. Han, H. Park, D. Kim, and B.-M. Kim, Opt Express 13, 5151 (2005).

11. D. Wang, Y. Chen, Y. Wang, and J. T. C. Liu, Opt. Lett. 38, 5280 (2013).

12. G. J. Tearney, R. H. Webb, and B. E. Bouma, Opt. Lett. 23, 1152 (1998).

13. A. Safrani and I. Abdulhalim, Opt. Lett. 37, 458 (2012).

14. M. G. Somekh, C. W. See, and J. Goh, Opt. Commun. 174, 75 (2000).

15. M. C. Pitter, C. W. See, and M. G. Somekh, Opt. Lett. 29 , 1200 (2004).

16. Y. Choi, T. D. Yang, K. J. Lee, and W. Choi, Opt. Lett. 36, 2465 (2011).

17. A. Dubois, L. Vabre, A.-C. Boccara, and E. Beaurepaire, Appl. Opt. 41, 805 (2002).

18. J.-F. Seurin, G. Xu, V. Khalfin, A. Miglo, J. D. Wynn, P. Pradhan, C. L. Ghosh, and L. A. D'Asaro, Proc. SPIE 7229, 722903 (2009)

19. A.-H. Dhalla, J. V. Migacz, and J. A. Izatt, Opt. Lett. 35, 2305 (2010).

20. L. Wang, T. Tschudi, T. Halldórsson, and P. R. Pétursson, Appl. Opt. 37, 1770 (1998).

21. A. Mermillod-Blondin, H. Mentzel, and A. Rosenfeld, Opt. Lett. 38, 4112 (2013).

22. T. Ikeda, G. Popescu, R. R. Dasari, and M. S. Feld, Opt. Lett. 30, 1165 (2005). 